\title{
A Novel Method for Calculating Luminance in the Access Zone of a Road Tunnel based on a Digital Camera
}

\author{
Wei He${ }^{1,2}$ and Bo Liang ${ }^{1, *}$ \\ ${ }^{1}$ School of Civil Engineering, Chongqing Jiaotong University, Chongqing 400074, China \\ ${ }^{2}$ Department of Traffic and Municipal Engineering, Sichuan College of Architectural Technology, Chengdu 610399, China
}

Received 1 January 2021; Accepted 7 May 2021

\begin{abstract}
Luminance $L 20$ in the access zone of a tunnel is affected by many factors, such as weather, latitude and longitude, time, and the type of scenery outside the tunnel. Currently available methods for obtaining $L 20$ exhibit shortcomings. To provide a low-cost, efficient, and highly accurate approach for determining $L 20$, a novel method for calculating $L 20$ was proposed in this study. A Nikon D7500 camera was calibrated using a spot-type luminance meter, and the point luminance formula was developed. The vignetting characteristics of the camera were analyzed when point luminance in the $20^{\circ}$ conical field of view $\left(20^{\circ}\right.$ field) was estimated using the formula. A MATLAB pseudocode was written to implement the proposed method for calculating L20. Field measurements verified the accuracy and efficiency of the proposed method. Results show that the proposed method for calculating $L 20$ can be applied to any digital single-lens reflex camera and road tunnel, and it exhibits a high degree of automation in post-data processing, increasing postprocessing efficiency by more than 10 times that of the traditional method. The proposed method eliminates the error of calculating $L 20$ by using point luminance, and it can improve the accuracy of $L 20$. That is, the higher the pixels of the RGB sensor of the metering system of a digital camera, the higher the accuracy of the $L 20$ estimated using the digital camera. The proposed method provides an excellent prospect for realizing intelligent tunnel lighting.
\end{abstract}

Keywords: Camera, L20, Luminance estimation, $20^{\circ}$ field

\section{Introduction}

The black hole effect of a tunnel is caused by the inability of the human eye to adapt to the dramatic change in luminance upon entering a tunnel. One of the measures for avoiding the black hole effect is to minimize luminance in the access zone of a tunnel, such as the large area with vegetation outside a tunnel and the dark materials used on the outer wall of a tunnel.[1] The International Commission on Illumination (CIE) recommended an equivalent veiling luminance or $L 20$ as the luminance in the access zone of tunnels. $L 20$ is the average luminance of a $20^{\circ}$ conical field of view $\left(20^{\circ}\right.$ field) when an observer situated at the starting point of an access zone looks toward a central point located at one-quarter of the tunnel portal height. $L 20$ represents the state of adaptation of the eye of a driver approaching the tunnel entrance. L2O is used as the basis for computing luminance in the entrance zone.[2] Thus, L20 is a critical parameter for the design of highway tunnel lighting systems. L20 considerably influences tunnel investment and daily lighting operating costs, as validated by using the Tokyo Bay Subsea Tunnel in Japan as an example. L20 was changed from $4000 \mathrm{~cd} / \mathrm{m}^{2}$ to $6000 \mathrm{~cd} / \mathrm{m}^{2}$, resulting in a $34 \%$ difference in equipment cost and a $30 \%$ difference in annual power consumption under the same conditions.[3]

Scholars have reduced the electricity consumption of tunnel lighting under the premise of improving traffic safety and comfort. Intelligent tunnel lighting that has emerged in recent years can adjust tunnel lighting in real time in

*E-mail address: liangbo_laoshi@163.com

ISSN: 1791-2377 @ 2021 School of Science, IHU. All rights reserved.

doi:10.25103/jestr.143.17 accordance with the luminance in the access zone of the tunnel. This process is increasingly demanding on the efficiency and accuracy of $L 20$ acquisition. The theoretical calculation of $L 20$ has become a considerable challenge to engineers because of numerous influencing factors, such as weather, latitude and longitude, time, and the type of scenery outside a tunnel.

In the existing literature, many studies have focused on test methods for L20.[4, 5] Considering that the luminance of all objects in the $20^{\circ}$ field cannot be obtained simultaneously, a point luminance meter is inefficient and exhibits poor accuracy in measuring L20. An imaging photometer has two significant drawbacks when measuring L20. First, the equipment is expensive; second, the test site requires a power supply. Given that they are low cost and efficient for testing, digital cameras are extensively used to test luminance distribution. However, post-data processing is inefficient, and the accuracy of the obtained L20 is poor. Currently available methods for acquiring $L 20$ are flawed. Therefore, obtaining L20 efficiently, accurately, and economically is a pressing issue.

Based on the preceding analysis, the current study improves the approach for calculating L20. Subsequently, the effects of the metering systems of digital cameras are also considered.

\section{State of the art}

Scholars have conducted considerable work using digital cameras to measure the distribution of scene luminance. Moore et al.[6] determined the luminance of surfaces within 
a scene through the relationship between luminosity and luminance; however, their proposed formula had a small range of luminance adaptation. Zatari et al.[7]pre-calibrated a CCD digital video camera mounted on a test vehicle to estimate the relationship between the gray value of light images and illumination parameters (luminance and illuminance); nevertheless, the operation was poorly automated. Kruisselbrink et al.[8]proposed choosing a camera resolution that can accurately measure luminance while minimizing privacy sensitivity and computational costs. However, a high resolution is required for measuring maximum luminance, and this condition can lead to automatic facial recognition and high computational costs. Suway et al.[9]proposed a method for calculating luminance by using a digital video camera. Their method allowed users to install a camera near the driver's eye and drive through a scene while capturing video. Still images from the video were then outputted and analyzed to form a luminance map; however, the suitability of the proposed formula for different camera sensors was not analyzed. Kruisselbrink et al.[10] presented the determination of luminance distribution by capturing high dynamic range (HDR) images and converting RGB information into CIE XYZ color space with slightly more insufficient accuracy between 5\% and 20\%. By using the Raspberry Pi computer and camera module as an absolute luminance sensor, Mead et al.[11] measured luminance values from $10 \mathrm{~cd} / \mathrm{m}^{2}$ to $50,000 \mathrm{~cd} / \mathrm{m}^{2}$ by adopting HDR imaging techniques with different shutter speeds in a sequence of images, enabling economical and accurate measurement of luminance distribution across a field of view. However, the camera used was costly to calibrate. Kayakuş et al.[12]developed artificial intelligenceand web-based software to perform road lighting measurements by using photographs of roads. With this software, road lighting measurements can be performed quickly, online, and without cost; however, the software was not validated on different roads with various lighting installations. Słomiński[13, 14] investigated potential measurement errors arising from the incompatibility of luminance measurement device parameters (i.e., number of pixels, sensor size, and focal length) with human eye resolution. Tian et al. [15]presented a calculation method for obtaining luminance parameters based on HDR image technology and concluded that the accuracy of the calculated target luminance values for all color blocks improved as background luminance decreased. However, the linear fitting of $I_{b}-X_{B}$ was low, and this problem may affect the accuracy of subsequent calculations. Song et al.[16] illustrated the principle of extracting luminance values from HDR images and demonstrated the feasibility of using such images in measurement methods for road light environment. Yi et al. [17] used a digital camera to obtain luminance distribution and concluded that luminance accuracy from the output image that used Adobe RGB color space was more accurate than the sRGB format, although the process was highly complicated. By using images captured with a digital camera, Sun et al. [18]calculated the luminance values of the scene at a tunnel portal but did not provide the shooting settings of the camera. Chuang [19]obtained the average luminance of a scene by using an image processing technology and a luminance detection algorithm. The results can be displayed and uploaded in real time to meet the detection requirements of tunnel luminance, but the system used an OV7670 camera with low pixels. Xiao[20]utilized an imaging photometer to calibrate an OV5640 camera and measured luminance in the access zone of a tunnel by using the calibrated camera. A Linux-based luminance measurement system was developed via programming, but the astigmatic lens was shaded in front of the dot matrix light-emitting diode light source to make the light source as evenly distributed as possible when the camera was calibrated. Guo et al. [21] established a method for measuring luminance in the access zone of a tunnel based on a digital imaging technology; however, this method did not address the issue of handling data errors.

The aforementioned studies have used digital cameras to measure the luminance values of scenes, but research that improves the method for calculating luminance $L 20$ in the access zone of a tunnel remains rare. The current work uses a point luminance meter to calibrate a consumer-grade digital camera (Nikon D7500) to develop the formula for point luminance. $L 20$ is obtained by calculating the average luminance of all the pixels in a $20^{\circ}$ field, and a MATLAB pseudocode is prepared to implement the proposed method. The vignetting characteristics of the camera are analyzed when estimating point luminance in the $20^{\circ}$ field by using the D7500 camera. The processing efficiency and accuracy of the proposed method in practical applications are verified. The effect of different metering systems of digital cameras on the error of the $L 20$ estimated using the proposed method is discussed.

The remainder of this study is organized as follows. Section 3 proposes the formula for point luminance and a novel method for calculating L20. Section 4 verifies the proposed method through measurements and discusses factors that affect the accuracy of L20. The final section summarizes the conclusions.

\section{Methodology}

\subsection{Formula for point luminance}

A digital camera should be used to estimate the point luminance for $L 20$ calculation. On the basis of geometrical optics, the pixel photometric exposure, $E$, is expressed as: [19]

$$
E=L \cdot \frac{\pi}{4} \cdot \frac{1}{F^{2}} \cdot \mu \cdot T \cdot(1-f / d)^{2}
$$

where $L$ is the point luminance of an object's surface $\left(\mathrm{cd} / \mathrm{m}^{2}\right), \pi$ is a constant (radians), $F$ is the aperture of the camera lens, $\mu$ is the optical transmittance of the camera lens, $T$ is the exposure time of the image (s), $f$ is the focal length of the camera lens $(\mathrm{mm})$, and $d$ is the distance between the camera lens and the object $(\mathrm{mm})$.

Given that $f / d$ is minimal and negligible, $E$ can also be expressed as:

$$
E=L \cdot \frac{\pi}{4} \cdot \frac{1}{F^{2}} \cdot \mu \cdot T
$$

Under normal exposure conditions, the pixel grayscale value is linearly related to the logarithm of the pixel photometric exposure, which can be expressed as: [5]

$G=v \cdot \lg E+m$ 
where $G$ is the pixel grayscale value, $v$ is the slope, $\lg E$ is the logarithm of the pixel photometric exposure, and $m$ is the intercepting element.

The D7500 camera (Nikon, Tokyo, Japan), equipped with an AF-P DX NIKKOR VR lens $18-55 \mathrm{~mm} \mathrm{f} / 3.5-5.6 \mathrm{G}$, was calibrated with a spot-type luminance meter (LS-150, Konica Minolta, Tokyo, Japan). The different camera settings used to scale the scene luminance captured by the camera sensor enabled the estimation of a diverse range of luminance values. [9] The camera settings during calibration are provided in Table 1.

Table 1. Settings of Nikon D7500 camera

\begin{tabular}{|c|c|c|c|}
\hline $\begin{array}{l}\text { Camera } \\
\text { parameter }\end{array}$ & Value & $\begin{array}{l}\text { Camera } \\
\text { parameter }\end{array}$ & Value \\
\hline Flash control & $\mathrm{M}$ & Flicker reduction & OFF \\
\hline $\begin{array}{l}\text { Chosen image } \\
\text { area }\end{array}$ & DX & $\begin{array}{l}\text { Remote control } \\
\text { mode (ML-L3) }\end{array}$ & OFF \\
\hline Image quality & $\mathrm{RAW}+\mathrm{F}^{\circ}$ & $\begin{array}{l}\text { Auto bracketing } \\
\text { set }\end{array}$ & OFF \\
\hline Image size & L & $\begin{array}{l}\text { Multiple } \\
\text { exposures }\end{array}$ & OFF \\
\hline $\begin{array}{l}\text { NEF (RAW) } \\
\text { compression }\end{array}$ & $\begin{array}{l}\text { Lossless } \\
\text { compressed }\end{array}$ & $\begin{array}{l}\text { Interval timer } \\
\text { shooting }\end{array}$ & OFF \\
\hline $\begin{array}{l}\text { NEF (RAW) bit } \\
\text { depth }\end{array}$ & 14 bit & Focus mode & AF-A \\
\hline ISO sensitivity & 100 & AF area mode & $\begin{array}{l}\text { Automatic } \\
\text { area } \mathrm{AF}\end{array}$ \\
\hline $\begin{array}{l}\text { Auto ISO } \\
\text { sensitivity } \\
\text { control }\end{array}$ & OFF & Metering & Matrix \\
\hline White balance & AUTO & Shooting mode & M \\
\hline $\begin{array}{l}\text { Set picture } \\
\text { control }\end{array}$ & NL & Focal length & $18 \mathrm{~mm}$ \\
\hline Color space & Adobe & $\begin{array}{l}\text { Optical shock } \\
\text { absorption }\end{array}$ & $\mathrm{ON}$ \\
\hline $\begin{array}{l}\text { Active D- } \\
\text { lighting }\end{array}$ & OFF & Release mode & S \\
\hline $\begin{array}{l}\text { Long exposure } \\
\text { NR }\end{array}$ & OFF & $\begin{array}{l}\text { Flash } \\
\text { compensation }\end{array}$ & 0 \\
\hline High ISO NR & OFF & $\begin{array}{l}\text { Exposure } \\
\text { compensation }\end{array}$ & 0 \\
\hline Vignette control & OFF & $\begin{array}{l}\text { Exposure } \\
\text { indication }\end{array}$ & $\begin{array}{l}\text { Good } \\
\text { exposure }\end{array}$ \\
\hline $\begin{array}{l}\text { Auto distortion } \\
\text { control }\end{array}$ & OFF & & \\
\hline
\end{tabular}

Abbreviations: M: Manual; DX: $23.5 \times 15.7 \mathrm{~mm}^{2}$ image area chosen to record images; RAW $+\mathrm{F}^{*}$ : Record two images, one raw image and one fine-quality JPEG image; NEF: Nikon electric film, which is another form of a RAW file format; ISO: International Organization for Standardization; NL: Natural; NR: Noise reduction; AF-A: Autofocusautomatic servo; AF: Autofocus; S: Single.

The following measures can be adopted to reduce calibration errors.

1) A card with color consistency should be used as the test target.

2) To eliminate the influence of viewing angle on luminance, the viewing direction of the camera and the spot luminance meter must be perpendicular to the surface of the card.

3) Outdoor luminance is dynamic, and thus, the luminance of the card should be measured with a spot luminance meter while simultaneously capturing images.

Different colored cards were used as the measured objects under clear and overcast conditions, and the cards were fixed at the center of the image. The calibration results are presented in Fig. 1.

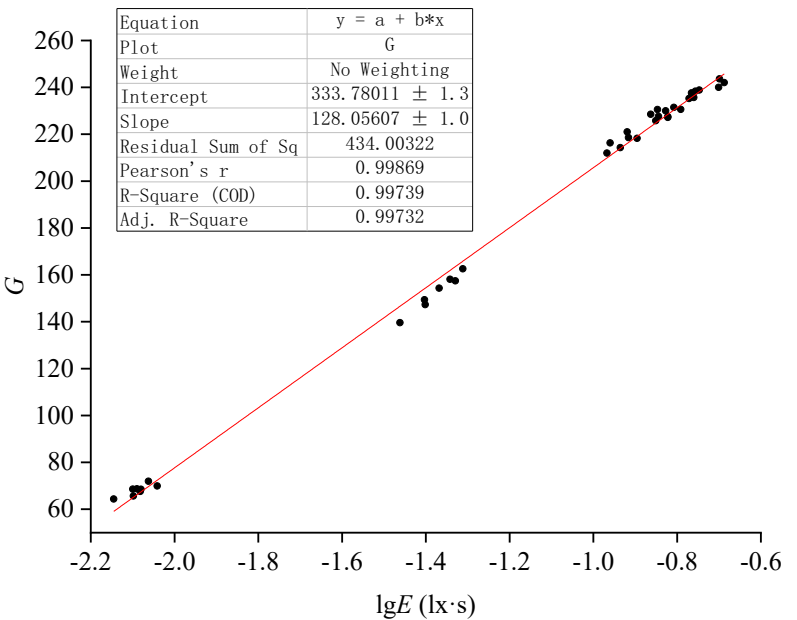

Fig. 1. Relationship between $G$ and $\lg E$ for the images captured by the Nikon D7500 camera using the settings in Table 1

As shown in Fig. 1, an excellent linear correlation exists between $G$ and $\lg E$ within the range of 6.05-10550 $\mathrm{cd} / \mathrm{m}^{2}$, which encompasses the luminance values of a typical daytime tunnel scene with a Pearson's r of 0.99869. The linear formula is as follows:

$$
G=128.06 \lg E+333.78
$$

On the basis of (2) and (4), (5) was derived for point luminance estimation by using the D7500 camera.

$$
L=5.305 \cdot \frac{F^{2}}{T} \cdot 10^{\frac{G-333.78}{128.06}}
$$

\subsection{Traditional method for estimating $L 20$}

L20 is typically calculated using the following formula recommended by CIE:

$$
L 20=\gamma \cdot L c+\rho \cdot L r+\varepsilon \cdot L e+\tau \cdot L t h
$$

where $\gamma$ is the percentage of the sky area in the $20^{\circ}$ field, $\rho$ is the percentage of the road area, $\varepsilon$ is the percentage of the surrounding area, $\tau$ is the percentage of the tunnel entrance area, $L c$ is the luminance of the sky, $L r$ is the luminance of the road, $L e$ is the luminance of the surroundings, and $L t h$ is the luminance of the tunnel entrance.

\subsection{Proposed method for estimating $L 20$}

\subsection{1 $20^{\circ}$ field grayscale image}

If the image acquired in accordance with the $L 20$ definition is converted into a grayscale image in MATLAB (R2016b, MathWorks, Natick, MA, USA), the area beyond the $20^{\circ}$ field on the grayscale image is covered with a black mask with a grayscale value of zero. In the $20^{\circ}$ field grayscale image shown in Fig. 2, the pixel grayscale value in the $20^{\circ}$ field is greater than zero and within the range of $1-255$. The pixel grayscale value beyond the $20^{\circ}$ field is equal to zero; therefore, MATLAB can immediately identify the $20^{\circ}$ field. 


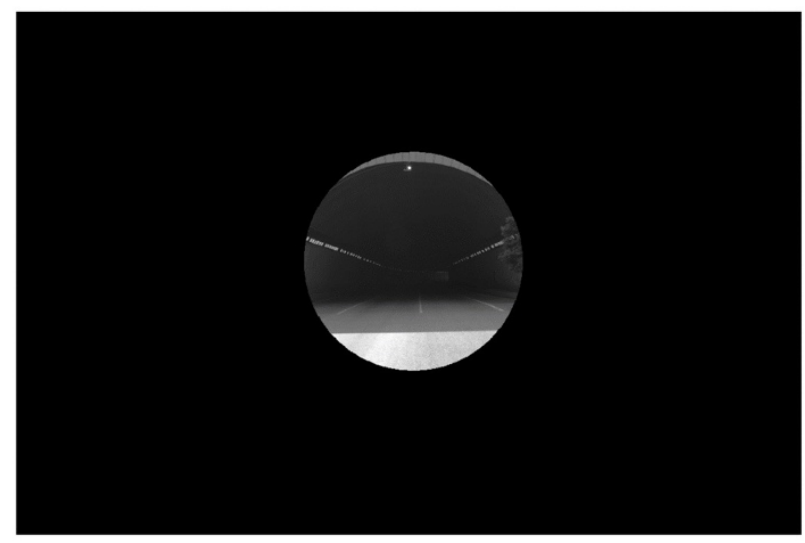

Fig. 2. $20^{\circ}$ field grayscale image

\subsubsection{Proposed $L 20$ calculation method}

$L 20$ is the average luminance of the $20^{\circ}$ field; hence, the proposed $L 20$ calculation method divides the sum of the luminance of all the pixel points in the $20^{\circ}$ field by the total number of pixels in the $20^{\circ}$ field to obtain L20. The following formula is adopted to calculate $L 20$ :

$$
L 20=\frac{\sum_{G=1}^{G=255} 5.305 \frac{F^{2}}{T} 10^{\frac{G-333.78}{128.06}} \operatorname{count}(G)}{\sum_{G=1}^{G=255} \operatorname{count}(G)}
$$

where count $(G)$ is the number of pixels with grayscale value $G$ in the $20^{\circ}$ field grayscale image.

\subsubsection{MATLAB pseudocode for implementing the proposed method}

First, a digital image is opened in the image viewer of MATLAB. Under the measure distance command, the tunnel vault is clicked to set the first point. Thereafter, the road directly below the vault is clicked to be selected as the second point. The results are exported to the workspace, and the first and second points are called "archtop" and "road," respectively, as shown in Fig. 3.

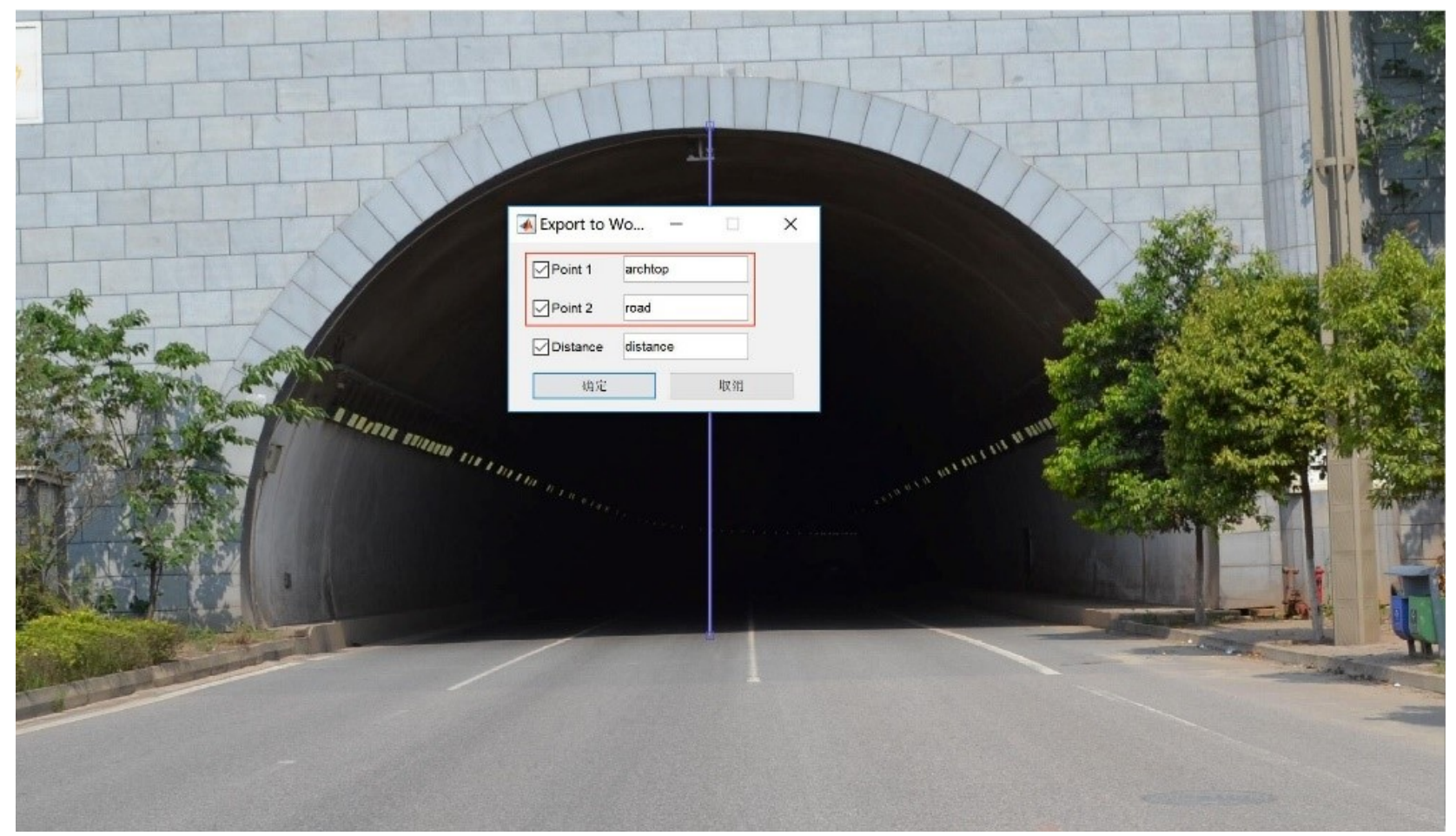

Fig. 3. Exporting the coordinates of the tunnel vault and the road directly below the vault to the workspace

Then, the following MATLAB pseudocode is executed.

\footnotetext{
Pseudocode: Obtain $L 20$ and the $20^{\circ}$ field grayscale images.

$1 H$ = input(' 'Height of tunnel portal(m):');

$2 S D=$ input( 'Stopping distance(m):');

3 savepath $=$ uigetdir(' $\left.\mathrm{C}: 1^{\prime}\right) ; \%$ Select the reading path of the image, the initial path is $\mathrm{C}: 1$

$4 x=\operatorname{archtop}(1) ; \%$ The abscissa $x$ of the center of the $20^{\circ}$ field in the image is obtained

$5 y=\operatorname{road}(2)-(\operatorname{road}(2)-\operatorname{archtop}(2)) / 4 ; \%$ The ordinate $y$ of the center of the $20^{\circ}$ field in the image is obtained

6 im $=\operatorname{dir}\left(\left[\right.\right.$ savepath, $\left.\left.{ }^{\prime *} . j p g '\right]\right) ; \%$ List all jpg format images in the savepath path and assign them to the $\boldsymbol{i m}$ array

7 imnumber $=$ length(im); \% imnumber is the number of jpg images in $\boldsymbol{i m}$ array

8 cimname $=$ cell $(1$, imnumber $) ; \%$ Define cimname as a cell array of 1 row, imnumber column

$9 \boldsymbol{V L 2 0}=[] ; \% \boldsymbol{V L 2 0}$ is defined as a row vector
} 
10 for $s=1:$ imnumber $\%$ Cycle from the first image to the imnumber-th image

11 imname $=\boldsymbol{i m}(s)$.name; \% Assign the s-th image name in the $\boldsymbol{i m}$ array to the variable imname

$12 \boldsymbol{i m r}=\operatorname{imread}\left(\left[\right.\right.$ savepath,,$^{\prime}$, imname $\left.]\right) ; \%$ Read the image named imname in the savepath path

13 cimname $\{s\}=$ imname; \% Assign imname to the s-th element of cimname array

14 imgray $=\operatorname{rgb} 2 \operatorname{gray}(\boldsymbol{i m r}) ; \%$ Get the gray image imgray

15 iminformation $=$ imfinfo([savepath,'’',imname $]) ; \%$ Assign the details of the image named imname in the savepath path to the iminformation array

$16 F=$ iminformation.DigitalCamera.FNumber; \% Assign the aperture to the variable $F$

$17 T=$ iminformation.DigitalCamera.ExposureTime; \% Assign the exposure time to the variable $T$

$18[\mathrm{im} W, \mathrm{im} H]=\operatorname{size}($ imgray $) ; \%$ Calculate the size of the grayscale image

$19 \boldsymbol{t}=\operatorname{linspace}(0,2 * \mathrm{pi}, 50) ; \% 0$ and $2 *$ pi are equally divided into 50 points, the row vector $\boldsymbol{t}$ with these 50 points is obtained

$20 r=(\operatorname{road}(2)-\operatorname{archtop}(2)) / H^{*} S D^{*} \operatorname{tand}(10) ; \%$ Assign the radius of $20^{\circ}$ field in the image to the variable $r$

21 immask $=\operatorname{poly} 2 \operatorname{mask}\left(r^{*} \cos (\boldsymbol{t})+x, r^{*} \sin (\boldsymbol{t})+y, \mathrm{im} W, \mathrm{im} H\right) ; \%$ mask covered beyond the $20^{\circ}$ field is assigned to the $\boldsymbol{i m m a s k}$ array

22 im 20withmask = immultiply(imgray,immask); \% Overlay the grayscale image and mask to get a $20^{\circ}$ field grayscale image and assign a

$20^{\circ}$ field grayscale image to the im20withmask array

23 Figure,imshow(im 20withmask); \% Show image named im 20withmask in the graphics dialog

24 saveas(gcf,[savepath,'`',imname(1:8),'gray','.png']); \% Save the current image in the savepath path and name the current image XXXXXXXXgray.png, XXXXXXXX are the first eight characters of the image name

25 [count,gray $]=\operatorname{imhist}($ im20withmask,256); \% Assign 256 levels of grayscale to the column vector gray and the number of pixels per grayscale to the column vector count

26 sumc $=0$

27 for $n=2: 256 \%$ Loop from grayscale 1 to grayscale 255

$28 s u m c=\operatorname{sumc}+\operatorname{count}(n) ; \%$ The total number of pixels in the $20^{\circ}$ field is assigned to the variable sumc

29 end

$30 \operatorname{sum} L=0$;

31 for $n=2: 256 \%$ Loop from grayscale 1 to grayscale 255

$32 \operatorname{sum} L=\operatorname{sum} L+5.305^{*} F^{\wedge} 2 / T^{*} \operatorname{power}(10,(\operatorname{gray}(n)-333.78) / 128.06) * \operatorname{count}(n) ; \%$ Assign the sum of the luminance of all pixels in the $20^{\circ}$ field to the variable sumL

33 end

$34 \boldsymbol{V L 2 0}(s)=s u m L /$ sumc; \% Assign the $L 20$ value of the s-th image to the variable $\boldsymbol{V L} 20(s)$

35 end

36 xlswrite([savepath,' \Results.xlsx'],cimname); \% Store the image names in the first line of Results.xlsx under the savepath path

37 xlswrite([savepath,' $\backslash$ Results.xlsx'],VL20,1,'A2'); \%Store the $L 20$ value in the second line of Results.xlsx under the savepath path

\section{Result Analysis and Discussion}

\subsection{Verifying the formula for point luminance through} measurements

The black and colored cards were located at the center and the edge of the $20^{\circ}$ field, respectively. The black card simulated the tunnel entrance, while the colored card simulated the scene outside the tunnel. Both point luminances were verified simultaneously in the $20^{\circ}$ field of an image, as shown in Fig. 4.

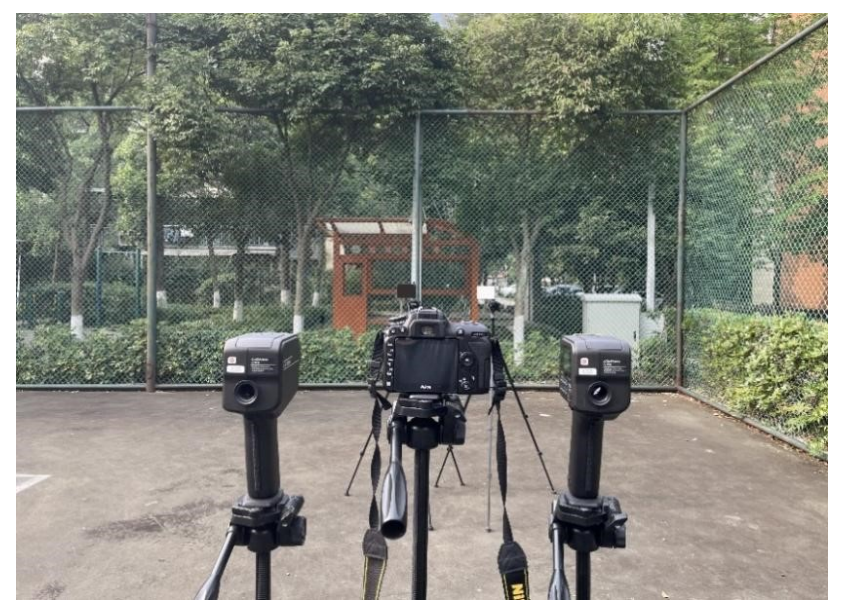

Fig. 4. Verification of point luminances
The point luminance estimated by (5) and that measured using the spot-type luminance meter (LS-150, Konica Minolta, Inc., Tokyo, Japan) were compared, and the results are presented in Table 2 and Fig. 5. Notably, all the estimated point luminances are close to the measured point luminances.

The histograms shown in Fig. 6 depict the frequency distribution of the percentage error expressed by (8). All point luminances fall within the $0-10 \%$ percentage error range.

Percentage error $=\frac{100 * \mid \text { estimated value }- \text { measured value } \mid}{\text { measured value }}$

Table 2 was further analyzed, and the results of the root-mean-square error (RMSE) and mean percentage error (MPE) are provided in Table 3.

$\mathrm{RMSE}=\sqrt{\frac{\sum(\text { estimated value }- \text { measured value })^{2}}{\text { data point number }}}$

$\mathrm{MPE}=\frac{\sum(100 * \mid \text { estimated value }- \text { measured value } / \text { measured value })}{\text { data point number }}(10)$ 
Table 2. Verification results of point luminance

\begin{tabular}{|c|c|c|c|c|c|c|}
\hline \multirow[b]{2}{*}{$\begin{array}{l}\text { Image } \\
\text { serial } \\
\text { number }\end{array}$} & \multicolumn{3}{|l|}{ Colored card } & \multicolumn{3}{|l|}{ Black card } \\
\hline & $\begin{array}{l}\text { Measured point } \\
\text { luminance }\left(\mathrm{cd} / \mathrm{m}^{2}\right)\end{array}$ & $\begin{array}{l}\text { Estimated point } \\
\text { luminance }\left(\mathrm{cd} / \mathrm{m}^{2}\right)\end{array}$ & $\begin{array}{l}\text { Percentage } \\
\text { error }(\%)\end{array}$ & $\begin{array}{l}\text { Measured point } \\
\text { luminance } \\
\left(\mathrm{cd} / \mathrm{m}^{2}\right)\end{array}$ & $\begin{array}{l}\text { Estimated point } \\
\text { luminance } \\
\left(\mathrm{cd} / \mathrm{m}^{2}\right)\end{array}$ & $\begin{array}{l}\text { Percentage } \\
\text { error }(\%)\end{array}$ \\
\hline 1 & 5502 & 5559.2 & 1 & 224.9 & 215.3 & 4.3 \\
\hline 2 & 820.1 & 744.5 & 9.2 & 135.3 & 124.1 & 8.2 \\
\hline 3 & 99.65 & 98.2 & 1.5 & 96.23 & 100.6 & 4.5 \\
\hline 4 & 1530 & 1649.3 & 7.8 & 71 & 77.8 & 9.6 \\
\hline 5 & 245.5 & 242.9 & 1.1 & 42.66 & 45.6 & 6.8 \\
\hline 6 & 30.85 & 28.5 & 7.5 & 29.88 & 29.5 & 1.3 \\
\hline 7 & 492.5 & 502.5 & 2 & 24.14 & 24.1 & 0.3 \\
\hline 8 & 131.8 & 127.5 & 3.3 & 22.68 & 22.7 & 0.2 \\
\hline 9 & 22.16 & 21.5 & 2.9 & 21.27 & 21.9 & 2.9 \\
\hline 10 & 571.6 & 614.3 & 7.5 & 27.78 & 28.5 & 2.5 \\
\hline 11 & 195.2 & 180.7 & 7.4 & 33.07 & 30 & 9.4 \\
\hline 12 & 49.39 & 45.7 & 7.4 & 47.09 & 44.9 & 4.7 \\
\hline 13 & 1301 & 1271.7 & 2.2 & 63.95 & 59.1 & 7.6 \\
\hline 14 & 447.2 & 402.6 & 10 & 73.06 & 66.6 & 8.9 \\
\hline 15 & 90.7 & 87.9 & 3.1 & 83.86 & 82.8 & 1.3 \\
\hline 16 & 2094 & 2247.3 & 7.3 & 92.44 & 92 & 0.5 \\
\hline 17 & 227.5 & 207.6 & 8.7 & 39.81 & 37.4 & 6.2 \\
\hline 18 & 35.86 & 32.5 & 9.4 & 34.91 & 33.5 & 4.1 \\
\hline 19 & 496.1 & 507.8 & 2.3 & 24.81 & 24.6 & 0.9 \\
\hline 20 & 134.8 & 131.5 & 2.5 & 23.6 & 22.8 & 3.3 \\
\hline 21 & 16.96 & 17 & 0.1 & 16.88 & 17.7 & 5 \\
\hline 22 & 291.7 & 291.9 & 0.1 & 14.4 & 14.4 & 0.2 \\
\hline 23 & 70.4 & 70.3 & 0.2 & 12.19 & 13.2 & 8.1 \\
\hline 24 & 11.32 & 10.6 & 6.5 & 11.13 & 11.9 & 6.6 \\
\hline
\end{tabular}

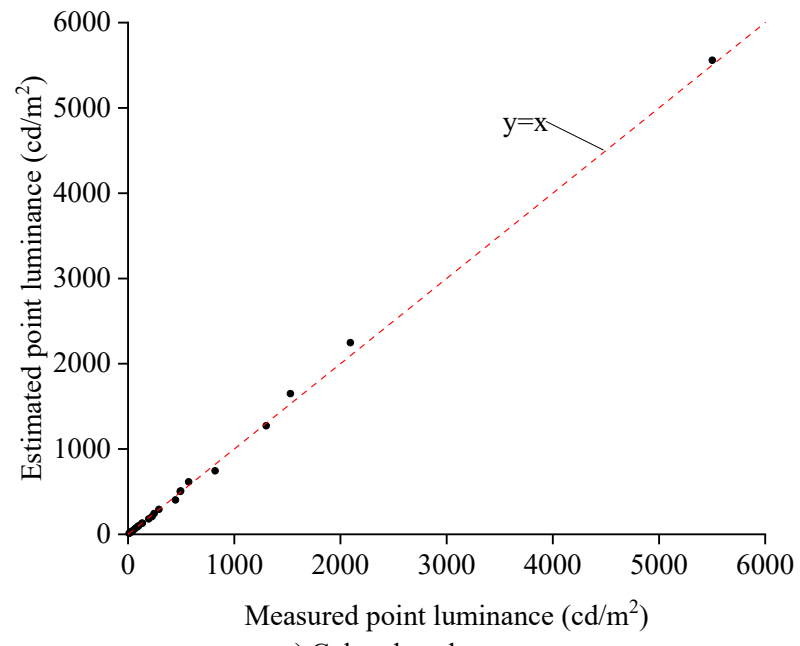

a) Colored card

Fig. 5. Scatterplot of the measured point luminance vs. the estimated point luminance

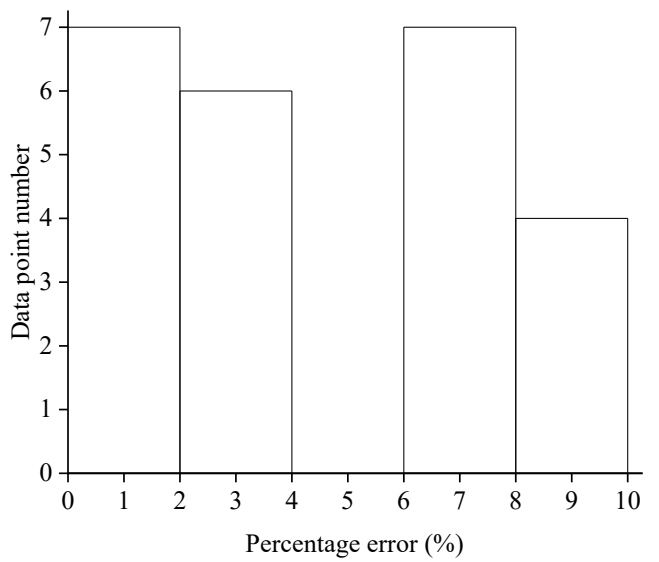

a) Colored card

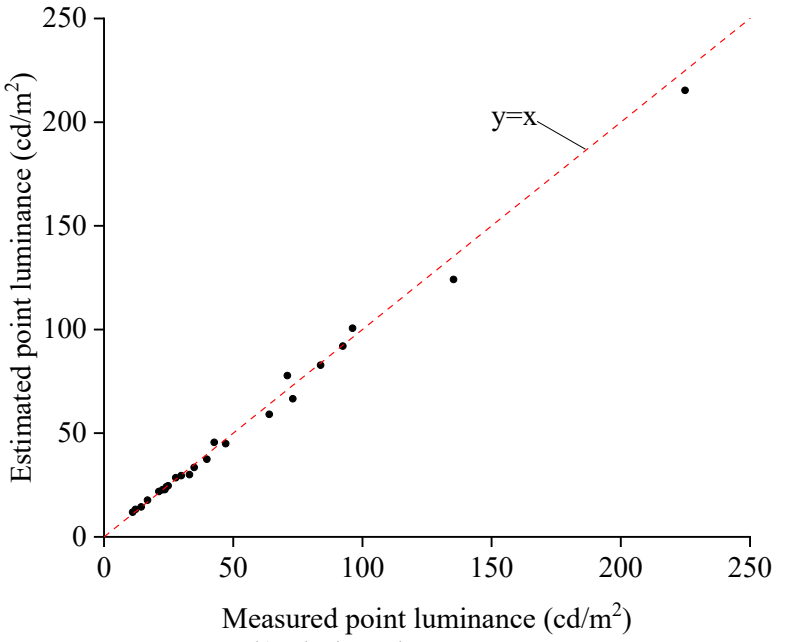

b) Black card

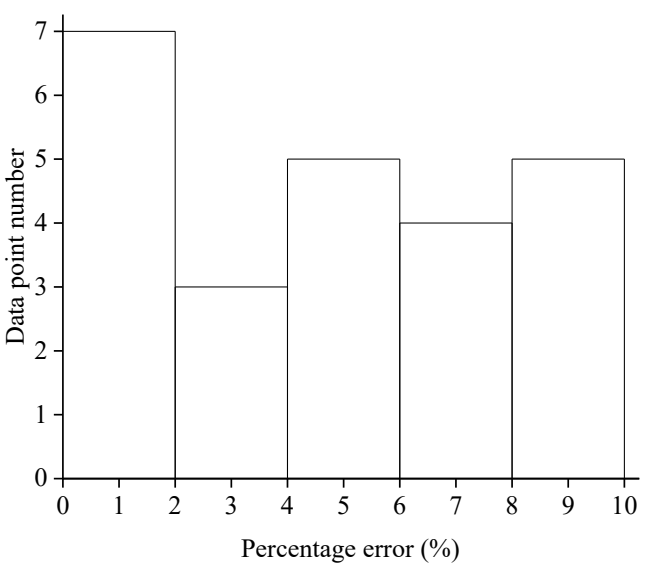

b) Black card

Fig. 6. Percentage error histogram of point luminance 
Table 3. RMSE and MPE comparison of the colored card vs. the black card

\begin{tabular}{l|l|l}
\hline & RMSE $\left(\mathrm{cd} / \mathrm{m}^{2}\right)$ & MPE (\%) \\
\hline $\begin{array}{l}\text { Point luminance on the } \\
\text { colored card }\end{array}$ & 46.7 & 4.6 \\
$\begin{array}{l}\text { Point luminance on the } \\
\text { black card }\end{array}$ & 4 & 4.5 \\
\hline
\end{tabular}

The colored card at the edge of the $20^{\circ}$ field and the black card at the center of the image have similar point luminance MPEs. The result shows that the D7500 camera exhibits no significant vignetting characteristic in the $20^{\circ}$ field, and the point luminance estimated by (5) does not require correction. The $20^{\circ}$ field is located in the central area of the image, accounting for $9 \%$ of the entire image area; thus, the vignetting characteristic can be disregarded. [22]Even when a significant difference exists between the two point luminances in the $20^{\circ}$ field of the same image (such as Images 1, 4, and 13), the percentage errors of the two point luminances estimated by (5) remain small. In summary, the D7500 camera can be used to estimate any point luminance in the $20^{\circ}$ field of the tunnel by using (5).

\subsection{Verifying the methods for estimating L20 via} measurements

In accordance with the definition of $L 20$, the D7500 camera was used to capture images of the portal of the Tiangongtang Tunnel in Deyang City, Sichuan Province, China. The L20 values estimated using the traditional method and the proposed method were compared with that measured using the imaging photometer (IC-PMY16, Radiant Vision Systems, Redmond, WA, USA). The results are presented in Fig. 7. Notably, the L20 estimated using the proposed method is closer to the measured L20 than that estimated using the traditional method.

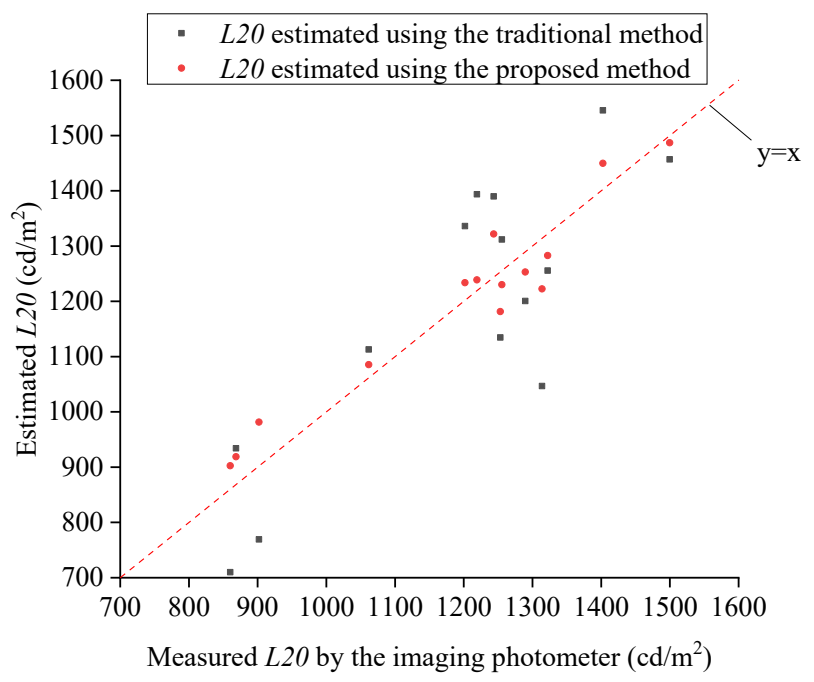

Fig. 7. Scatterplot of the measured $L 20$ vs. the estimated $L 20$

The percentage error histogram of $L 20$ is shown in Fig. 8. The percentage error ranges of $L 20$ estimated using the traditional and proposed methods are $2 \%-22 \%$ and $0-10 \%$, respectively.

The RMSE and MPE of the $L 20$ values estimated using the traditional and proposed methods are provided in Table 4. The RMSE and MPE of the L20 estimated using the proposed method are smaller than those of the $L 20$ estimated using the traditional method, indicating that the L2O estimated using the proposed method is more accurate than the one estimated using the traditional method.
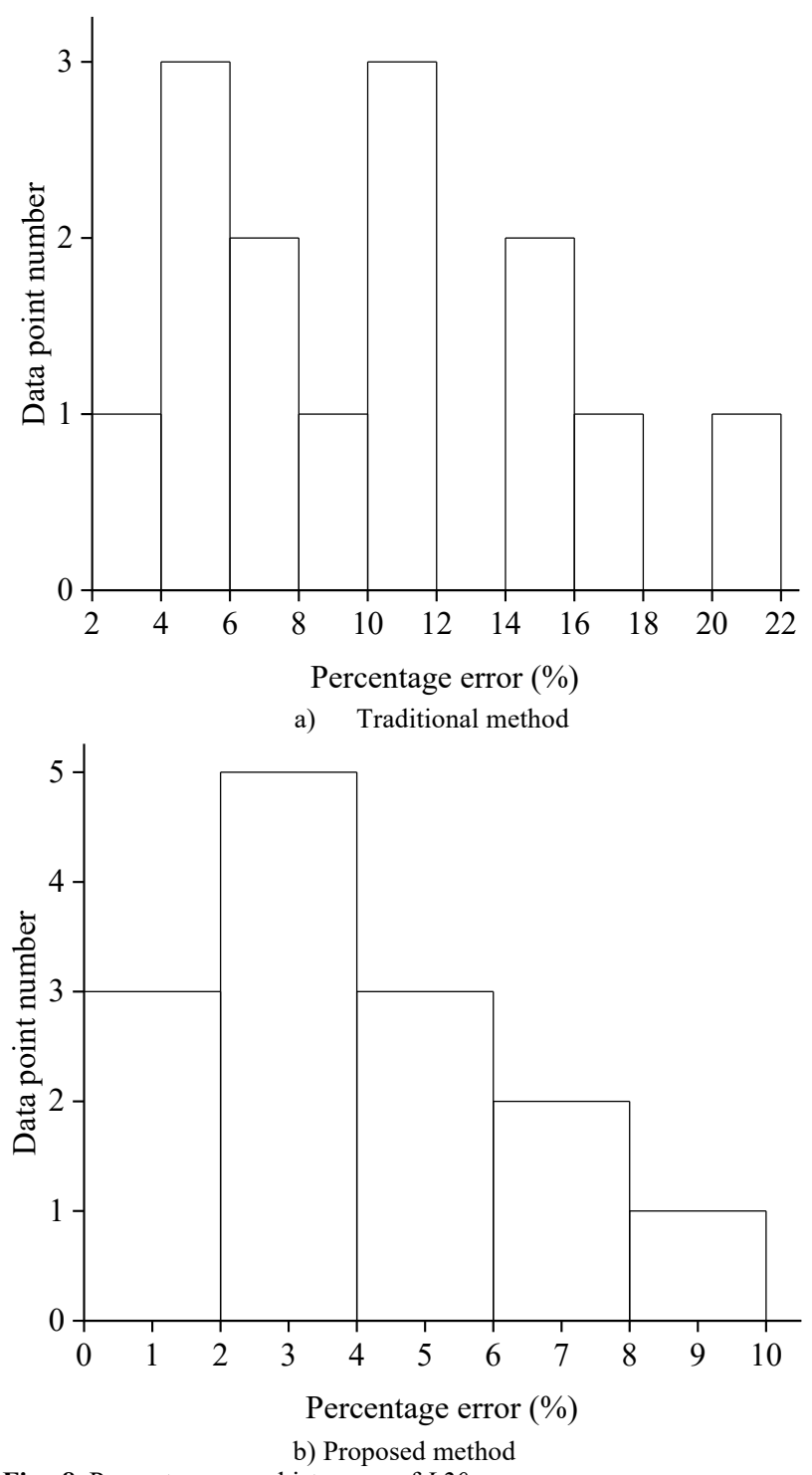

Fig. 8. Percentage error histogram of $L 20$

Table 4. RMSE and MPE comparison of the traditional method vs. the proposed method

\begin{tabular}{l|l|l}
\hline & RMSE $\left(\mathrm{cd} / \mathrm{m}^{2}\right)$ & MPE (\%) \\
\hline $\begin{array}{l}\text { L20 estimated using the } \\
\text { traditional method } \\
\begin{array}{l}L 20 \text { estimated using the } \\
\text { proposed method }\end{array}\end{array}$ & 131 & 10.1 \\
\hline
\end{tabular}

4.3 Comparison of the traditional and proposed methods for estimating $L 20$

Point luminance on an object's surface is estimated using (5) for both post-data processing methods. However, L20 is calculated differently. The traditional method takes the mean luminance of three points on the object as the approximate object luminance and obtains the approximate L2O in accordance with (6). The error of the traditional method has two sources: the error in estimating point luminance by using (5) and the error in estimating $L 20$ by using point luminance. The proposed method divides the total luminance of all the pixels in the $20^{\circ}$ field by the total number of pixels in the $20^{\circ}$ field to obtain the average luminance $L 20$. The proposed method is accurate in calculating $L 20$ by using point luminance; therefore, the proposed method has only one source of error, i.e., the error arising from estimating 
point luminance by using (5). Moreover, the proposed method eliminates the need to calculate the percentage of object area in the $20^{\circ}$ field.

The traditional method is time-consuming and inefficient when estimating the $L 20$ of the same tunnel portal at different times. The proposed method is only required to input the coordinates of the tunnel vault and the road directly below the vault, the height of the tunnel portal, stopping distance, and the paths of the images. The 220 values and $20^{\circ}$ field grayscale images can be obtained quickly. Presenting the Tiangongtang Tunnel as a case study, the traditional method takes $20 \mathrm{~min}$ to obtain the $L 20$ for the first image and $3 \mathrm{~min}$ for each subsequent image. When the proposed method is used, the $L 20$ of the first image can be obtained within 2 min and each subsequent image can be taken in less than $3 \mathrm{~s}$. The proposed method is more than 10 times faster than the traditional method in obtaining the $L 20$ of a tunnel portal for a specific moment. The speed advantage of the proposed method is more apparent when dealing with the $L 20$ of the same tunnel entrance at multiple moments.

\subsection{Factors that affect the accuracy of the $L 20$ estimated} using the proposed method

The accuracy of point luminance directly determines the accuracy of L20 when using the proposed method, and different metering systems affect the estimated accuracy of point luminance. The lens of the Nikon D3500 camera is an AF-S DX NIKKOR VR 18-55 mm f/3.5-5.6 G. The point luminance formula for the Nikon D3500 camera, shown in (11), applies the settings in Table 5.

$$
L=5.305 \cdot \frac{F^{2}}{T} \cdot 10^{\frac{G-324.03}{125.93}}
$$

Table 5. Settings of the Nikon D3500 camera

\begin{tabular}{|c|c|c|c|}
\hline $\begin{array}{c}\text { Camera } \\
\text { parameter }\end{array}$ & Value & $\begin{array}{c}\text { Camera } \\
\text { parameter }\end{array}$ & Value \\
\hline Image quality & RAW+F & Focus mode & AF-A \\
\hline Image size & $\mathrm{L}$ & $\mathrm{AF}$ area mode & $\begin{array}{c}\text { Automatic area } \\
\mathrm{AF}\end{array}$ \\
\hline ISO sensitivity & 100 & $\begin{array}{l}\text { Built-in AF } \\
\text { auxiliary } \\
\text { illuminator }\end{array}$ & OFF \\
\hline $\begin{array}{c}\text { Auto ISO } \\
\text { sensitivity } \\
\text { control }\end{array}$ & OFF & Metering & Matrix \\
\hline White balance & AUTO & $\begin{array}{l}\text { Flash control } \\
\text { built-in flash }\end{array}$ & $\mathrm{M}$ \\
\hline $\begin{array}{l}\text { Set picture } \\
\text { control }\end{array}$ & NL & $\begin{array}{c}\text { Flash } \\
\text { compensation }\end{array}$ & 0 \\
\hline Color space & Adobe & $\begin{array}{l}\text { Exposure } \\
\text { compensation }\end{array}$ & 0 \\
\hline $\begin{array}{l}\text { Active D- } \\
\text { lighting }\end{array}$ & OFF & $\begin{array}{l}\text { Exposure } \\
\text { indication }\end{array}$ & Good exposure \\
\hline NR & OFF & Shooting mode & M \\
\hline Vignette control & OFF & Focal length & $18 \mathrm{~mm}$ \\
\hline $\begin{array}{l}\text { Auto distortion } \\
\text { control }\end{array}$ & OFF & $\begin{array}{l}\text { Optical shock } \\
\text { absorption }\end{array}$ & $\mathrm{ON}$ \\
\hline
\end{tabular}

The point luminances estimated using the D3500 and D7500 cameras were compared with that measured by the Konica Minolta LS-150 luminance meter. The results are presented in Fig. 9. The estimated point luminance is close to the measured point luminance.

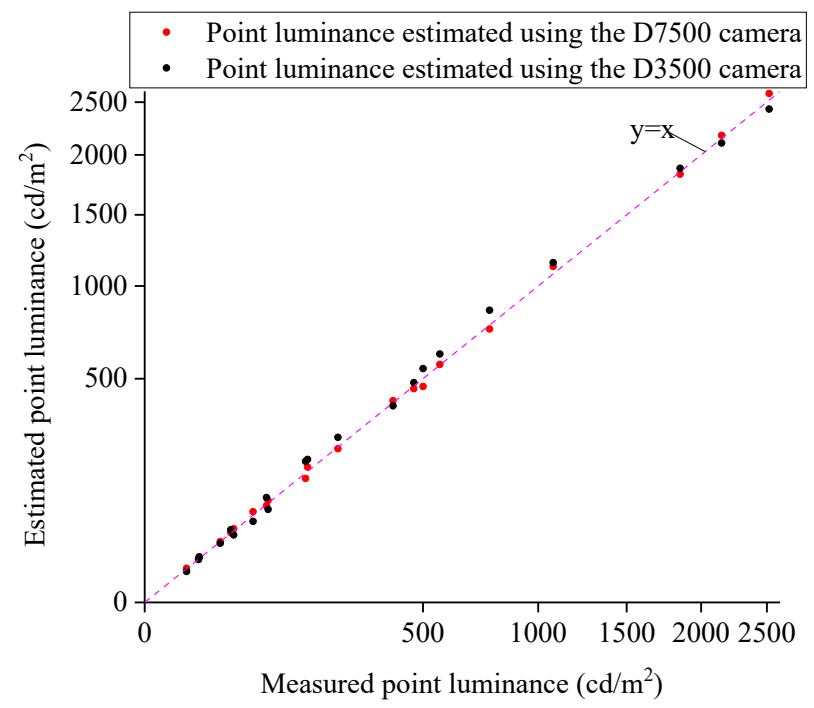

Fig. 9. Scatterplot of the measured point luminance vs. the point luminance estimated using the D7500 and D3500 cameras

The percentage error histogram of point luminance is shown in Fig. 10. The percentage error ranges of point luminance estimated using the D7500 and D3500 cameras are $0-10 \%$ and $0-20 \%$, respectively.

The RMSE and MPE of the point luminance estimated using the D7500 and D3500 cameras are provided in Table 6 . The RMSE and MPE of the point luminance estimated using the D7500 camera are smaller than those of the point luminance estimated using the D3500 camera. The D7500 camera provides point luminance with higher accuracy because its metering system uses an RGB sensor with higher pixels, as indicated in Table 7. The D7500 camera estimates $L 20$ with better accuracy than the D3500 camera because the only source of error in estimating $L 20$ in the proposed method is point luminance error. Compared with the D3500 camera, the D7500 camera exhibits a $10 \%$ reduction in the maximum percentage error when estimating $L 20$.

Table 6. RMSE and MPE comparison of the D7500 camera vs. the D3500 camera

\begin{tabular}{l|c|c}
\hline & RMSE $\left(\mathrm{cd} / \mathrm{m}^{2}\right)$ & MPE $(\%)$ \\
\hline $\begin{array}{l}\text { Point luminance } \\
\text { estimated using the } \\
\text { D7500 camera }\end{array}$ & 27.4 & 5.4 \\
$\begin{array}{l}\text { Point luminance } \\
\text { estimated using the } \\
\text { D3500 camera }\end{array}$ & 47.6 & 13.8 \\
\hline
\end{tabular}

Table 7. Comparison of the metering systems of the D7500 and D3500 cameras

\begin{tabular}{l|l|l}
\hline \multicolumn{1}{c|}{$\begin{array}{c}\text { Technical } \\
\text { Specification }\end{array}$} & \multicolumn{1}{c}{ D3500 camera } & \multicolumn{1}{c}{ D7500 camera } \\
\hline $\begin{array}{l}\text { Exposure } \\
\text { metering system }\end{array}$ & $\begin{array}{l}\text { TTL exposure } \\
\text { metering using a } \\
420 \text { pixel RGB } \\
\text { sensor }\end{array}$ & $\begin{array}{l}\text { TTL exposure metering } \\
\text { using an RGB sensor with } \\
\text { approximately 180,000 } \\
\text { pixels }\end{array}$ \\
\hline
\end{tabular}

TTL: Through the lens 

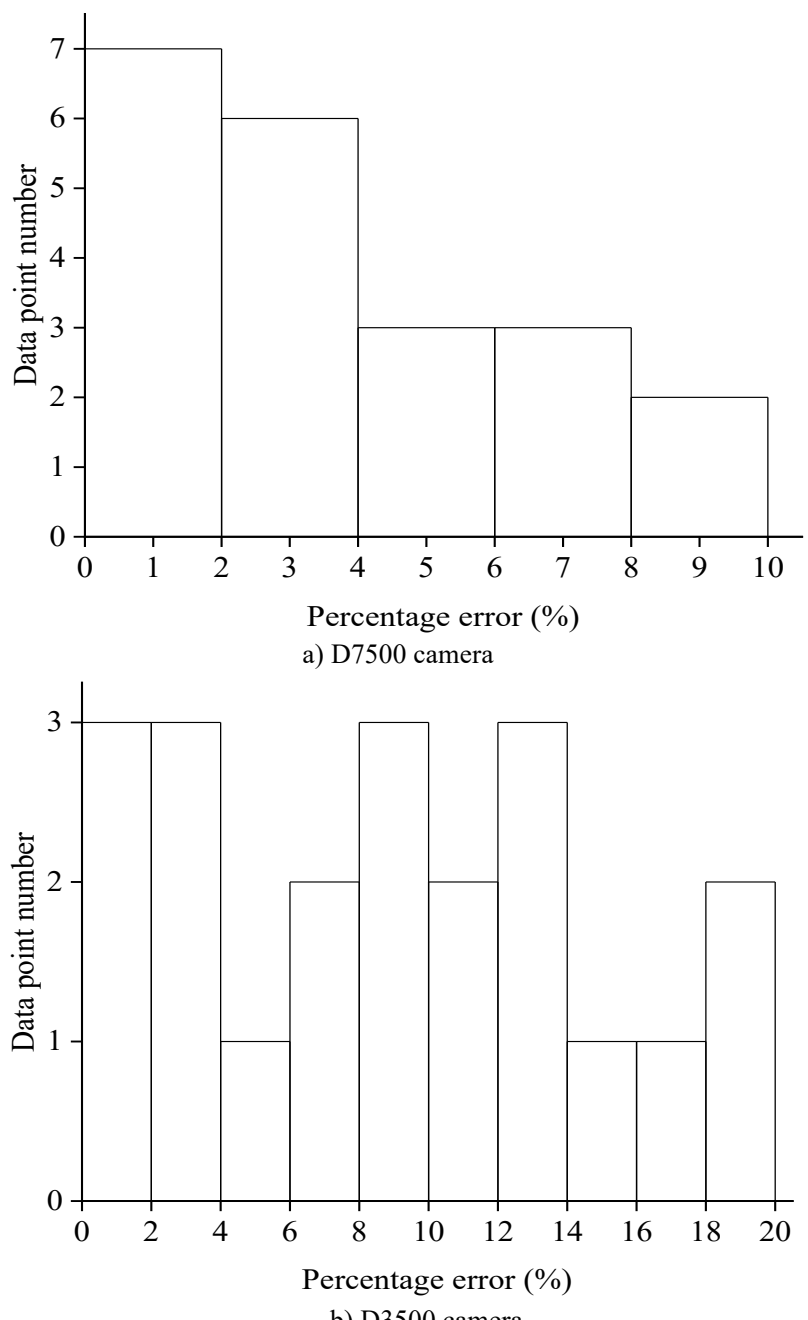

Fig. 10. Percentage error histogram of point luminance

\section{Conclusion}

To obtain $L 20$ efficiently, accurately, and economically, this study calculated $L 20$ by dividing the sum of the luminance of all the pixels in the $20^{\circ}$ field by the total number of pixels in the $20^{\circ}$ field. Moreover, a pseudocode was written in MATLAB to obtain $L 20$ and $20^{\circ}$ field grayscale images. The following conclusions were drawn.

(1) Any digital single-lens reflex (DSLR) camera can be calibrated in accordance with the procedure described in the current study to develop a formula for estimating point luminance. The formula adapts to a wide range of luminance and can be used to estimate any point luminance within a $20^{\circ}$ field of a tunnel without correction.

(2) The proposed method for calculating $L 20$ can be applied to any DSLR camera and road tunnel. The proposed method exhibits a high degree of automation in post-data processing, significantly improving post-processing efficiency. The proposed method for calculating $L 20$ eliminates the error of $L 20$ calculated using point luminance and improves the accuracy of $L 20$.

(3) The higher the pixels of the RGB sensor of the metering system of a digital camera, the higher the accuracy of the $L 20$ estimated using the digital camera.

This study combined field measurements with theoretical work and proposed a novel method for calculating L20. The proposed method achieves balance among efficiency, accuracy, and cost. Moreover, the method contributes to the realization of intelligent tunnel lighting. Considering the lack of an evenly distributed integrating sphere as a light source, the use of an integrating sphere to calibrate a digital camera in future studies can provide more accurate $L 20$.

\section{Acknowledgements}

This work was supported by the National Natural Science Foundation of China (Grant No. 51878107).

This is an Open Access article distributed under the terms of the Creative Commons Attribution License.

\section{References}

1. Onaygil, S., Güler, Ö.,Erkin, E., "Determination of the effects of structural properties on tunnel lighting with examples from Turkey". Tunnelling and underground space technology, 18(1), 2003, pp.85-91.

2. l'éclairage, C. i. d., "Guide for the lighting of road tunnels and underpasses". Vienna: C. C. Bureau, Austria, 2004, pp.26-27.

3. China Merchants Chongqing Communications Research and Design Institute Co., L., "Guidelines for Design of Lighting of Highway Tunnels ". Beijing: China Communications Press Co., Ltd, China, 2014, pp.15-16.

4. Qu, Z., Zhao, Q.,Liu, X., "Tunnel Ambient Exterior Luminance L20 (S) Tested by Environmental Sketch Method". Technology of Highway and Transport, 1(5), 2004, pp.117-121.

5. TU, Y., WANG, S., HOU, L., ZHANG, Q., CAI, J., XIE, X., LIU, Q.,PAN, Y., "Research on Access Zone Luminance of Expressway Tunnel in Southwest Mountainous Areas of Zhejiang Province". Highway, (1), 2012, pp.198-203.

6. Moore, T., Graves, H., Perry, M.,Carter, D., "Approximate field measurement of surface luminance using a digital camera". Lighting Research \& Technology, 32(1), 2000, pp.1-11.

7. Zatari, A., Dodds, G., McMenemy, K.,Robinson, R., "Glare, luminance, and illuminance measurements of road lighting using vehicle mounted CCD cameras". Leukos, 1(2), 2005, pp.85-106.

8. Kruisselbrink, T. W., Dangol, R.,van Loenen, E. J., "Recommendations for long-term luminance distribution measurements: the spatial resolution". Building and Environment, 169, 2020, 106538.

9. Suway, J. A.,Suway, S., "A Method for Digital Video Camera Calibration for Luminance Estimation". Austria, 2017.

10. Kruisselbrink, T., Aries, M.,Rosemann, A., "A practical device for measuring the luminance distribution". International Journal of Sustainable Lighting, 19(1), 2017, pp.75-90.

11. Mead, A. R.,Mosalam, K. M., "Ubiquitous luminance sensing using the Raspberry Pi and Camera Module system". Lighting Research \& Technology, 49(7), 2017, pp.904-921.

12. Kayakus, M.,Üncü, I., "Research note: the measurement of road lighting with developed artificial intelligence software". Lighting Research \& Technology, 51(6), 2019, pp.969-977.

13. Słomiński, S., "Identifying problems with luminaire luminance measurements for discomfort glare analysis". Lighting Research \& Technology, 48(5), 2016, pp.573-588.

14. Słomiński, S., "Typical Causes of Errors during Measuring Luminance Distributions in Relation to Glare Calculations". In: 2018 VII. Lighting Conference of the Visegrad Countries (Lumen V4), Trebic, Czech Republic: IEEE, 2018, pp.1-5.

15. Tian, H., Hao, T., Zhang, H.,Niu, P., "Luminance Measurement Based on High Dynamic Range Images". Laser \& Optoelectronics Progress, 56(6), 2019, pp.061502.

16. Song, J., Wang, L.,Chen, Y., "The Principles and Methods to Measuring Road Lighting by High Dynamic Image Technology". China Illuminating Engineering Journal, 26(2), 2015, pp.21-23,46. 
17. Yi, B., Zhen, J., Yuan, T.,Mi, H., "Selection of digital cameras' color space conversion matrix as an imaging luminance measurement device". Journal of Electronic Measurement and Instrumention, 29(03), 2015, pp.427-432.

18. Sun, c., Chen, Z., Yang, C., "Research on Environment Luminance at Tunnel Portal with Digital Camera". China Illuminating Engineering Journal, 21(6), 2010, pp.19-22,27.

19. Chuang, J., "Study on Tunnel Luminance Detection System Based on Image Processing". Master thesis of Xi'an University of Architecture and Technology, China, 2012, pp.53-54.

20. Xiao, Q., "Measurement of Tunnel Luminance and Vehicle Detection Based on Video Stream and its Application". Master thesis of Guilin university of electronic technology, China, 2018, pp.16-33.

21. Guo, C., Liu, Y., Wang, M., Sun, Z., Zhang, X.,Shen, J., "Analytical Methods of the Road Tunnel Ambient Exterior Luminance Using Automatic Digital Test Technology". China Illuminating Engineering Journal, 25(05), 2014, pp.87-92.

22. Bellia, L., Cesarano, A., Minichiello, F., Sibilio, S.,Spada, G., "Calibration procedures of a CCD camera for photometric measurements". In: Instrumentation and Measurement Technology Conference, Vail, CO, USA: IEEE, 2003, pp.89-93. 